\title{
COVID-19 patients with hypertension have more severe disease: a multicenter retrospective observational study
}

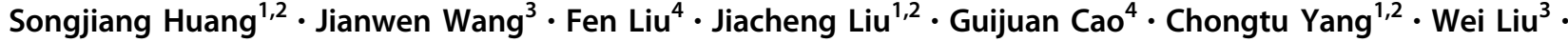 \\ Chao $\mathrm{Tu}^{5} \cdot$ Muxin $\mathrm{Zhu}^{3} \cdot$ Bin Xiong ${ }^{1,2}$
}

Received: 30 April 2020 / Revised: 8 May 2020 / Accepted: 8 May 2020 / Published online: 1 June 2020

(C) The Japanese Society of Hypertension 2020

\begin{abstract}
This study aims to explore the effect of hypertension on disease progression and prognosis in patients with coronavirus disease 2019 (COVID-19). A total of 310 patients diagnosed with COVID-19 were studied. A comparison was made between two groups of patients, those with hypertension and those without hypertension. Their demographic data, clinical manifestations, laboratory indicators, and treatment methods were collected and analyzed. A total of 310 patients, including 113 patients with hypertension and 197 patients without hypertension, were included in the analysis. Compared with patients without hypertension, patients with hypertension were older, were more likely to have diabetes and cerebrovascular disease, and were more likely to be transferred to the intensive care unit. The neutrophil count and lactate dehydrogenase, fibrinogen, and D-dimer levels in hypertensive patients were significantly higher than those in nonhypertensive patients $(P<0.05)$. However, multivariate analysis (adjusted for age and sex) failed to show that hypertension was an independent risk factor for COVID-19 mortality or severity. COVID-19 patients with hypertension were more likely than patients without hypertension to have severe pneumonia, excessive inflammatory reactions, organ and tissue damage, and deterioration of the disease. Patients with hypertension should be given additional attention to prevent worsening of their condition.
\end{abstract}

Keywords Coexistent disease $\cdot$ Hypertension $\cdot$ Prognosis $\cdot$ SARS-COV-2 $\cdot 2019$ novel coronavirus disease

These authors contributed equally: Songjiang Huang, Jianwen Wang, Fen Liu

\author{
Bin Xiong \\ herr_xiong@126.com
}

1 Department of Radiology, Union Hospital, Tongji Medical College, Huazhong University of Science and Technology, Wuhan, China

2 Hubei Province Key Laboratory of Molecular Imaging, Wuhan, China

3 Tuberculosis and Respiratory Department, Wuhan Jinyintan Hospital, Wuhan, China

4 Department of Radiology, the Central Hospital of Wuhan, Tongji Medical College, Huazhong University of Science and Technology, Wuhan, Hubei, China

5 Department of Critical Care Medicine, Wuhan Jinyintan Hospital, Wuhan, Hubei, China

\section{Introduction}

At the end of 2019, a series of unexplained pneumonia cases appeared and showed a tendency for transmission from person to person. Soon, scientists isolated the causative pathogen, a new type of $\beta$ coronavirus [1], which was subsequently named by the International Committee on Taxonomy of Viruses as Severe acute respiratory syndrome coronavirus 2 (SARS-COV-2) [2]. The disease was named coronavirus disease 2019 (COVID-19) by the World Health Organization [3]. As of April 19, 2020, the cumulative number of confirmed cases worldwide was 2,242,395, and the cumulative death toll was 152,551 [4].

Angiotensin-converting enzyme 2 (ACE2) is considered to be the receptor for SARS-COV-2 to enter human cells [5]. ACE2 is widely expressed in various organ systems of the human body, including the respiratory system and the cardiovascular system [6]. The enzyme can catalyze the conversion of angiotensin II (Ang II) to angiotensin 1-7, which is a peptide that counteracts the proinflammatory, prooxidation, and vasoconstrictive effects of Ang II. Studies have shown that hypertension may be one of the most 
common comorbid diseases in patients with COVID-19 [7]. A study involving 1099 COVID-19 patients had a prevalence of hypertension of $23.4 \%$ in severe patients [8]. Because of the interaction between SARS-COV-2 and ACE2 and the role of ACE2 in the pathogenesis of hypertension, there are many speculations that hypertension may be involved in the pathogenesis of COVID-19. However, some early reports have not revealed whether there is a relationship between hypertension and disease severity. Therefore, it remains unclear whether high blood pressure is a risk factor for COVID-19 or whether blood pressure control in hypertensive patients with COVID-19 is a lowrisk factor.

To understand whether hypertension is a risk factor affecting the progress and prognosis of COVID-19, this study included 310 cases of COVID-19 patients admitted to Wuhan Jinyintan Hospital and the Central Hospital of Wuhan after the outbreak in 2020. Their demographic data, clinical manifestations, laboratory indicators, and treatment methods were collected and analyzed.

\section{Methods}

\section{Participants}

In this study, we collected the clinical data of 310 patients diagnosed with COVID-19 at Wuhan Jinyintan Hospital and the Central Hospital of Wuhan after the outbreak in 2020. According to the World Health Organization's temporary guidelines, all the patients enrolled in this study were diagnosed with COVID-19. In the first part of the study, all patients were divided into two groups according to whether they had high blood pressure. To avoid the effects of other complications, the second part of the study excluded patients with complications other than hypertension and then divided the remaining patients into a hypertension group and a nonhypertension group. We monitored all patients in our study and recorded the entire course of the disease and the final clinical outcome of each person.

\section{Data sources}

The demographic data, epidemiological characteristics, clinical features, severity of the disease, laboratory tests and treatment results of the patients were obtained from the medical record system and recorded. The date of onset was defined as the date of the first onset of symptoms.

\section{Definition}

All patients with COVID-19 met the following criteria: (1) epidemic history, (2) fever or other respiratory symptoms,
(3) abnormal CT imaging of typical viral pneumonia, and (4) positive SARS-COV-2 RNA by RT-PCR. According to their medical history, the patients were divided into a hypertension group and a nonhypertension group.

The clinical classification of patients was based mainly on the novel coronavirus pneumonia diagnosis and treatment plan (trial version 7) developed by the National Health Committee of the People's Republic of China [9]. Classifications could be summarized as follows: (1) mild type: only mild clinical symptoms with no sign of pneumonia in imaging features; (2) common type: complicated with fever, respiratory symptoms and imaging features of pneumonia; (3) severe type: complicated with any of the following: (a) respiratory distress, respiratory rate $\geq 30$ beats/min; (b) mean oxygen saturation $\leq 93 \%$ at rest; or (c) ratio of the partial pressure of arterial oxygen to the fraction of inspired oxygen $\left(\mathrm{PaO}_{2}: \mathrm{FiO}_{2}\right) \leq 300 \mathrm{mmHg}(1 \mathrm{mmHg}=0.133 \mathrm{kPa})$; and (4) critical type: complicated with any of the following: (a) respiratory failure and mechanical ventilation required; (b) shock; or (c) organ dysfunction and intensive care unit admission required. In our research, we combined both the severe type and critical type patients into the severe group.

\section{Statistical analysis}

Using SPSS25.0 software (IBM, Inc., Chicago, IL, USA), the categorical variables are expressed as frequencies (percentages), and the continuous variables are expressed as the mean \pm standard deviation or the median of the quartile interval whenever appropriate. Student's $t$ test or Mann-Whitney U test was used for continuous variables, and chi-square test or Fisher exact test was used for categorical variables. A multivariable logistic regression model (adjusted for age and sex) was used in a multivariate analysis. A $P$ value $<0.05$ was considered statistically significant.

\section{Results}

Of the 310 patients with COVID-19, the median age was 62 years (interquartile range, 49-70). The number of women was $136(43.9 \%)$, and the number of men was $174(56.1 \%)$. The most common symptom of all patients was fever, which was observed in $81.9 \%$ of the patients. The frequencies of other symptoms were cough $(63.2 \%)$, dyspnea $(52.3 \%)$, expectoration (19.4\%), muscle ache $(7.7 \%)$, and diarrhea $(12.9 \%)$, whereas headache occurred in 3 cases (1.0\%). The most common complications were hypertension $(36.5 \%)$ and diabetes $(15.5 \%)$, followed by cerebrovascular diseases $(6.8 \%)$ and cardiovascular diseases $(6.1 \%)$. The number of deaths in the hospital was 58 (18.7\%). A total of 155 patients $(50 \%)$ were in the severe group (including severe type and critically ill type, 
according to our definition). Compared with nonhypertensive patients, hypertensive patients were older (67 [IQR, 58-73] vs 57 [IQR, 40-68]), had higher rates of diabetes $(28.3 \%$ vs $8.1 \%)$, were more commonly complicated with cerebrovascular diseases $(12.4 \%$ vs $3.6 \%)$, had more dyspnea $(61.1 \%$ vs $47.2 \%)$, and had fewer cough symptoms $(55.8 \%$ vs $67.5 \%)$. Compared with nonhypertensive patients, hypertensive patients had a higher mortality rate $(24.8 \%$ vs $15.2 \%)$, a higher proportion of severe patients $(63.7 \%$ vs $42.1 \%)$, a higher proportion of patients receiving noninvasive mechanical ventilation (16.8\% vs $7.6 \%$ ), and a higher proportion of patients transferred to the intensive care unit (ICU) $(23.9 \%$ vs 12.2\%) (Table 1).
The laboratory indexes of all patients (Table 2) showed lymphocytopenia (0.84 IQR, [0.58-1.21]), decreased albumin (33.9 IQR, [29.7-37.6]), increased lactate dehydrogenase (LDH) (289 IQR, [206-420.5]), increased erythrocyte sedimentation rate (ESR) (46.7 IQR, [28.75-63.00]), increased C-reactive protein (14.65 IQR, [3.14-73.80]), increased serum ferritin (785.57 IQR, [398.74-1290.13]), increased interleukin-6 (IL-6) (9.91 IQR, [6.61-14.44]), and increased D-dimer (0.735 IQR, [0.46-2.38]) in patients with COVID-19.

In addition, Table 2 also shows that patients with hypertension had significantly higher neutrophil counts (7.91 IQR, [3.41-7.98] vs 2.58 IQR, [1.90-6.92]), neutrophil-lymphocyte ratio (NLR) (9.98 IQR,
Table 1 Baseline characteristics and treatments of patients infected with SARS-CoV-2

\begin{tabular}{|c|c|c|c|c|}
\hline & $\begin{array}{l}\text { Total } \\
(n=310)\end{array}$ & $\begin{array}{l}\text { Nonhyperten- } \\
\text { sion } \\
(n=197)\end{array}$ & $\begin{array}{l}\text { Hypertension } \\
(n=113)\end{array}$ & $P$ value \\
\hline Age, years & $62(40-70)$ & $57(40-68)$ & $67(58-73)$ & $<0.001$ \\
\hline Sex & - & - & - & 0.395 \\
\hline Female & $136(43.9 \%)$ & $90(45.7 \%)$ & $46(40.7 \%)$ & \\
\hline Male & $174(56.1 \%)$ & $107(54.3 \%)$ & $67(69.3 \%)$ & \\
\hline \multicolumn{5}{|l|}{ Comorbidities } \\
\hline Constructive pulmonary disease & $9(2.9 \%)$ & $6(3.0 \%)$ & $3(2.7 \%)$ & 1.000 \\
\hline Diabetes mellitus & $48(15.5 \%)$ & $16(8.1 \%)$ & $32(28.3 \%)$ & $<0.001$ \\
\hline Cerebrovascular disease & $21(6.8 \%)$ & $7(3.6 \%)$ & $14(12.4 \%)$ & 0.003 \\
\hline Cardiovascular disease & $19(6.1 \%)$ & $11(5.6 \%)$ & $8(7.1 \%)$ & 0.597 \\
\hline Chronic liver disease & $4(1.3 \%)$ & $2(1.0 \%)$ & $2(1.8 \%)$ & 0.965 \\
\hline Cancer & $1(0.3 \%)$ & $1(0.5 \%)$ & $0(0.0 \%)$ & 1.000 \\
\hline \multicolumn{5}{|l|}{ Signs and Symptoms } \\
\hline Fever & $254(81.9 \%)$ & $163(82.7 \%)$ & $91(80.5 \%)$ & 0.626 \\
\hline Cough & $196(63.2 \%)$ & $133(67.5 \%)$ & $63(55.8 \%)$ & 0.039 \\
\hline Dyspnea & $162(52.3 \%)$ & $93(47.2 \%)$ & $69(61.1 \%)$ & 0.019 \\
\hline Expectoration & $60(19.4 \%)$ & $44(22.3 \%)$ & $16(14.2 \%)$ & 0.079 \\
\hline Muscle ache & $24(7.7 \%)$ & $13(6.6 \%)$ & $11(9.7 \%)$ & 0.320 \\
\hline Diarrhea & $40(12.9 \%)$ & $25(12.7 \%)$ & $15(13.3 \%)$ & 0.883 \\
\hline Headache & $3(1.0 \%)$ & $2(1.0 \%)$ & $1(0.9 \%)$ & 1.000 \\
\hline \multicolumn{5}{|l|}{ Treatments and prognosis } \\
\hline $\begin{array}{l}\text { Noninvasive mechanical } \\
\text { ventilation }\end{array}$ & $37(11.9 \%)$ & $21(10.7 \%)$ & $16(14.2 \%)$ & 0.360 \\
\hline Invasive mechanical ventilation & $34(11.0 \%)$ & $15(7.6 \%)$ & $19(16.8 \%)$ & 0.013 \\
\hline ECMO & $5(1.6 \%)$ & $1(0.5 \%)$ & $4(3.5 \%)$ & 0.116 \\
\hline ICU admission & $51(16.5 \%)$ & $24(12.2 \%)$ & $27(23.9 \%)$ & 0.007 \\
\hline $\begin{array}{l}\text { Duration of viral shedding after } \\
\text { COVID-19 onset, days }\end{array}$ & $7(5-12)$ & $8(8-11)$ & $7(5-13)$ & 0.144 \\
\hline Disease severity & - & - & - & \\
\hline Non-severe & $155(50.0 \%)$ & $114(57.9 \%)$ & $41(36.3 \%)$ & \\
\hline Severe & $155(50.0 \%)$ & $83(42.1 \%)$ & $72(63.7 \%)$ & $<0.001$ \\
\hline Death & $58(18.7 \%)$ & $30(15.2 \%)$ & $28(24.8 \%)$ & 0.038 \\
\hline Hospitalization time, days & $17(11-26)$ & $11(10-23)$ & $11(10-21)$ & 0.014 \\
\hline
\end{tabular}

ECMO extracorporeal membrane oxygenation, $I C U$ intensive care unit 
Table 2 Laboratory parameters of patients infected with SARS-CoV-2

\begin{tabular}{|c|c|c|c|c|c|}
\hline & Normal range & Total $(n=310)$ & Nonhypertension $(n=197)$ & Hypertension $(n=113)$ & $P$ value \\
\hline \multicolumn{6}{|l|}{ Laboratory indices } \\
\hline WBC, $\times 10^{9} / \mathrm{L}$ & $4.00-10.00$ & $5.53(4.06-8.36)$ & $3.30(2.62-8.70)$ & $7.52(4.66-9.04)$ & $<0.001$ \\
\hline $\mathrm{NC}, \times 10^{9} / \mathrm{L}$ & $1.80-6.30$ & $4.36(2.76-6.80)$ & $2.58(1.90-6.92)$ & $7.91(3.41-7.98)$ & $<0.001$ \\
\hline $\mathrm{LC}, \times 10^{9} / \mathrm{L}$ & $1.10-3.20$ & $0.84(0.58-1.21)$ & $0.56(0.49-1.42)$ & $0.73(0.49-0.90)$ & 0.366 \\
\hline NLR & $<5.87$ & $5.09(2.65-10.06)$ & $4.96(1.92-10.99)$ & $9.98(3.61-17.60)$ & 0.001 \\
\hline $\mathrm{SaO}_{2}, \%$ & $>95.0$ & $95.7(91.0-98.0)$ & $95.7(92.3-97.7)$ & $96.6(88.6-97.3)$ & 0.036 \\
\hline $\mathrm{TB}, \mu \mathrm{mol} / \mathrm{L}$ & $5.10-19.00$ & $11.20(8.50-15.50)$ & $12.10(8.85-13.20)$ & $10.70(10.25-14.60)$ & 0.162 \\
\hline ALT, U/L & $0-40$ & $27(17-49)$ & $26(11-34)$ & $57(31-113)$ & 0.007 \\
\hline AST, U/L & $0-40$ & $33.0(23.0-50.6)$ & $33.0(32.5-40.5)$ & $52.0(30.5-76.0)$ & 0.083 \\
\hline Albumin, g/L & $34.0-54.0$ & $33.9(29.7-37.6)$ & $28.3(27.6-34.1)$ & $31.1(29.7-35.6)$ & 0.002 \\
\hline Globulin, g/L & $20.0-35.0$ & $31.0(26.7-34.8)$ & $30.9(26.0-37.4)$ & $29.6(28.0-32.2)$ & 0.034 \\
\hline $\mathrm{LDH}, \mathrm{U} / \mathrm{L}$ & $109-245$ & $289(206-421)$ & $295(233-374)$ & $325(245-409)$ & 0.120 \\
\hline Creatinine, $\mu \mathrm{mol} / \mathrm{L}$ & $50.00-120.00$ & $69.50(56.70-85.38)$ & $66.70(52.05-86.95)$ & $72.30(55.45-84.85)$ & 0.009 \\
\hline $\mathrm{ESR}, \mathrm{mm} / \mathrm{h}$ & $<15.0$ & $46.7(28.8-63.0)$ & $58.0(21.5-61.0)$ & $43.0(29.5-63.5)$ & 0.104 \\
\hline $\mathrm{CRP}, \mathrm{mg} / \mathrm{L}$ & $<8.0$ & $14.7(3.1-73.8)$ & $44.3(20.8-112.2)$ & $35.3(13.9-91.7)$ & 0.001 \\
\hline Serum ferritin, $\mathrm{ng} / \mathrm{mL}$ & $21.80-275.0$ & $787.57(398.74-1290.13)$ & $831.72(503.48-1337.51)$ & $680.05(382.78-1356.80)$ & 0.218 \\
\hline IL-6, pg/mL & $0.10-2.90$ & $9.91(6.61-14.44)$ & $7.76(4.44-26.33)$ & $10.22(7.27-15.17)$ & 0.467 \\
\hline BNP, $\mathrm{pg} / \mathrm{mL}$ & $0.0-100.0$ & $67.1(28.0-165.2)$ & $55.3(38.3-200.9)$ & $72.5(28.9-82.7)$ & 0.438 \\
\hline D-dimer, ug/mL & $<0.50$ & $0.74(0.46-2.38)$ & $0.83(0.54-1.96)$ & $0.69(0.45-1.62)$ & 0.012 \\
\hline FIB, g/L & $2.00-4.00$ & $3.55(2.81-4.80)$ & $3.90(3.20-5.30)$ & $4.10(3.45-6.30)$ & 0.026 \\
\hline PT, s & $11.0-16.0$ & $11.4(10.6-12.3)$ & $11.5(10.4-12.2)$ & $11.1(10.0-12.2)$ & 0.060 \\
\hline APTT, s & $27.0-45.0$ & $28.1(24.8-32.7)$ & $29.3(23.7-33.1)$ & $23.4(20.6-30.5)$ & 0.004 \\
\hline
\end{tabular}

WBC white blood cell count, $N C$ neutrophil count, $L C$ lymphocyte count, $N L R$ neutrophil-to-lymphocyte ratio; $\mathrm{SaO} 2$ oxyhemoglobin saturation, $T B$ total bilirubin, $A L T$, alanine aminotransferase, $A S T$ aspartate aminotransferase, $L D H$ lactate dehydrogenase, ESR erythrocyte sedimentation rate, $C R P$ c-reactive protein, $I L-6$ interleukin-6, BNP b-type natriuretic peptide, $F I B$ fibrinogen, $P T$ prothrombin time, $A P T T$ activated partial thromboplastin time

[3.61-17.60] vs 4.96 IQR, [1.92-10.99]), alanine transaminase (ALT) (57 IQR, [21-113] vs 26 IQR [11-34],), creatinine (72.3 IQR, [55.45-84.85] vs 66.7 IQR, [52.05-86.95]) and fibrinogen (4.1 IQR, [3.45-6.3] vs 3.9 IQR, [3.2-5.3]) than those without hypertension. In addition, the activated partial prothrombin time (APTT) (23.4 IQR, [20.6-30.5] vs 29.3 IQR, [23.7-33.1]) in hypertensive patients was significantly lower than that in nonhypertensive patients. These data suggested that COVID-19 patients with hypertension tended to show more severe inflammation and organ damage than COVID-19 patients without hypertension, which may lead to a poor prognosis.

To better explore the impact of hypertension on the progression and prognosis of COVID-19 and avoid the confounding effects of other complications, in the following analysis, we excluded patients with complications other than hypertension, and the results are shown in Tables 3 and 4. Compared with nonhypertensive patients, hypertensive patients were older (66 IQR, [56-71] vs 54 IQR, [38-67]), had a higher proportion of severe cases $(64.6 \%$ vs $38.1 \%)$, and had a higher proportion of ICU admission (21.5\% vs
$11.3 \%)$. However, in terms of mortality and the length of hospital stay, there were no significant differences between the two groups of patients.

In addition, with regard to laboratory indexes, the white blood cell count (7.36 IQR, [4.86-9.73] vs 3.25 IQR, [2.34-9.51]), neutrophil count (7.92 IQR, [3.43-8.86] vs 2.52 IQR, [1.62-8.63]), NLR (14.96 IQR, [3.61-20.23] vs 5.15 IQR, [3.03-13.81]), ALT (46.5 IQR, [22.3-204.8] vs $20 \mathrm{IQR}$, [9.5-26.0]), LDH (316 IQR, [245-412] vs 302 IQR, [250-407]), D-dimer (1.09 IQR, [0.56-3.48] vs 0.74 IQR, [0.48-2.06]) and FIB (4.5 IQR, [3.8-6.3] vs 4.4 IQR, [3.5-5.6]) in hypertensive patients were significantly higher than those in nonhypertensive patients, while blood oxygen saturation (SaO2) (94.80 IQR, [81.98-96.73] vs 95.35 IQR, [91.53-98.05]) and APTT (22.3 IQR, [19.2-32.2] vs 30.8 IQR, [26.5-33.6]) in hypertensive patients were significantly lower than those in nonhypertensive patients.

In a multivariate analysis (Table 5), we separately used the severity or mortality of COVID-19 as dependent variables and age, sex, and hypertension as candidate independent variables to establish a multivariable logistic 
Table 3 Baseline characteristics and treatments of hypertensive and non- hypertensive COVID19 patients without other comorbidities

\begin{tabular}{|c|c|c|c|c|}
\hline & $\begin{array}{l}\text { Total } \\
(n=225)\end{array}$ & $\begin{array}{l}\text { Nonhypertension } \\
(n=160)\end{array}$ & $\begin{array}{l}\text { Hypertension } \\
(n=65)\end{array}$ & $P$ value \\
\hline Age, years & $59(45-68)$ & $54(38-67)$ & $66(56-71)$ & $<0.001$ \\
\hline Sex & - & - & - & 0.728 \\
\hline Female & $101(44.9 \%)$ & $73(45.6 \%)$ & $28(43.1 \%)$ & \\
\hline Male & $124(55.1 \%)$ & $87(54.4 \%)$ & $37(56.9 \%)$ & \\
\hline \multicolumn{5}{|l|}{ Signs and symptoms } \\
\hline Fever & $190(84.4 \%)$ & $136(85 \%)$ & $54(83.1 \%)$ & 0.718 \\
\hline Cough & $143(63.6 \%)$ & $108(67.5 \%)$ & $35(53.8 \%)$ & 0.054 \\
\hline Dyspnea & $113(50.2 \%)$ & $76(47.5 \%)$ & $37(56.9 \%)$ & 0.200 \\
\hline Expectoration & $39(17.3 \%)$ & $32(20.0 \%)$ & $7(10.8 \%)$ & 0.097 \\
\hline Muscle ache & $19(8.4 \%)$ & $12(7.5 \%)$ & $7(10.8 \%)$ & 0.424 \\
\hline Diarrhea & $24(10.7 \%)$ & $13(8.1 \%)$ & $11(16.9 \%)$ & 0.053 \\
\hline Headache & $2(0.9 \%)$ & $1(0.6 \%)$ & $1(1.5 \%)$ & 0.495 \\
\hline \multicolumn{5}{|l|}{ Treatments and prognosis } \\
\hline $\begin{array}{l}\text { Noninvasive mechanical } \\
\text { ventilation }\end{array}$ & $25(11.1 \%)$ & $16(10.0 \%)$ & $9(13.8 \%)$ & 0.405 \\
\hline $\begin{array}{l}\text { Invasive mechanical } \\
\text { ventilation }\end{array}$ & $22(9.8 \%)$ & $12(7.5 \%)$ & $10(15.4 \%)$ & 0.071 \\
\hline ECMO & $3(1.3 \%)$ & $1(0.6 \%)$ & $2(3.1 \%)$ & 0.201 \\
\hline ICU admission & $32(14.2 \%)$ & $18(11.3 \%)$ & $14(21.5 \%)$ & 0.045 \\
\hline $\begin{array}{l}\text { Duration of viral shedding } \\
\text { after COVID-19 onset, days }\end{array}$ & $8.0(6.0-12.0)$ & $9.0(8.0-11.5)$ & $8.5(5.0-14.0)$ & 0.044 \\
\hline Disease severity & - & - & - & \\
\hline Non-severe & $122(54.2 \%)$ & $99(61.9 \%)$ & $23(35.4 \%)$ & \\
\hline Severe & $103(45.8 \%)$ & $61(38.1 \%)$ & $42(64.6 \%)$ & $<0.001$ \\
\hline Death & $37(16.4 \%)$ & $23(14.4 \%)$ & $14(21.5 \%)$ & 0.189 \\
\hline Hospitalization time, days & $18.00(12.00-26.00)$ & $14.00(10.25-25.25)$ & $13.50(10.50-22.75)$ & 0.409 \\
\hline
\end{tabular}

$E C M O$ extracorporeal membrane oxygenation, $I C U$ intensive care unit regression model. The results showed that the odds ratios of hypertension were 1.562 (95\% CI: $0.929-2.625 ; P=0.092)$ and 1.262 (95\% CI: $0.683-2.332 ; P=0.458)$ in the analysis of severity and mortality, respectively.

Finally, we discussed the effects of other complications on the prognosis of hypertensive COVID-19 patients. We found that most of the laboratory indexes of hypertensive COVID-19 patients with other complications were not significantly different from those of hypertensive patients without other complications (Table 6), which suggested that the effect of other complications on the prognosis of hypertensive COVID-19 patients may be limited.

\section{Discussion}

In our study, the median age of COVID-19 patients was 62 years, and the prevalence rate of hypertension was $36.5 \%$. A national survey conducted in China from October 2012 to December 2015 showed that the total prevalence of hypertension in China's adult population ( $\geq 18$ years old) is
$23.2 \%$ [10]. In addition, a previous multicenter study showed that $30 \%$ of COVID-19 patients with a median age of 56 had previously coexisting hypertension; [2] another large sample of 1099 COVID-19 cases (with a median age of 47) had a prevalence of hypertension of $15 \%$ [8]. The higher prevalence of hypertension in our study may be explained by a higher median age. In general, there is not sufficient evidence to show that people with high blood pressure are more likely to be infected with COVID-19 than those without hypertension.

Our study revealed that hypertensive COVID-19 patients tend to show higher mortality. Previous clinical studies on SARS and Middle East respiratory syndrome showed that hypertension is a risk factor for increased mortality in infected patients $[11,12]$. The specific pathogenesis of hypertension that may lead to more severe COVID-19 remains to be studied. The imbalance of cytokines may be considered an explanation for the correlation between hypertension and severe COVID-19. Increasing clinical data have shown a relationship between the deterioration of COVID-19 and cytokine storms [7], such as elevated levels 
Table 4 Laboratory parameters of hypertensive and non- hypertensive COVID-19 patients without other comorbidities

\begin{tabular}{|c|c|c|c|c|c|}
\hline & Normal range & Total $(n=225)$ & Nonhypertension $(n=160)$ & Hypertension $(n=65)$ & $P$ value \\
\hline \multicolumn{6}{|l|}{ Laboratory indices } \\
\hline WBC, $\times 10^{9} / \mathrm{L}$ & $4.00-10.00$ & $5.23(3.90-7.67)$ & $3.25(2.34-9.51)$ & $7.36(4.86-9.73)$ & 0.002 \\
\hline $\mathrm{NC}, \times 10^{9} / \mathrm{L}$ & $1.80-6.30$ & $3.79(2.59-6.18)$ & $2.52(1.62-8.63)$ & $7.92(3.43-8.86)$ & 0.003 \\
\hline $\mathrm{LC}, \times 10^{9} / \mathrm{L}$ & $1.10-3.20$ & $0.86(0.59-1.19)$ & $0.54(0.48-0.62)$ & $0.65(0.40-0.85)$ & 0.624 \\
\hline NLR & $<5.87$ & $4.63(2.48-8.44)$ & $5.15(3.03-13.81)$ & $14.96(3.6-20.23)$ & 0.042 \\
\hline $\mathrm{SaO}_{2}, \%$ & $>95.0$ & $96.0(91.3-98.0)$ & $95.4(91.5-98.1)$ & $94.8(82.0-96.7)$ & 0.001 \\
\hline $\mathrm{TB}, \mu \mathrm{mol} / \mathrm{L}$ & $5.10-19.00$ & $11.15(8.48-15.10)$ & $10.55(8.78-13.00)$ & $10.60(9.75-13.40)$ & 0.375 \\
\hline ALT, U/L & $<40.0$ & $27.4(17.9-48.0)$ & $20.0(9.50-26.0)$ & $46.5(22.3-204.8)$ & 0.001 \\
\hline $\mathrm{AST}, \mathrm{U} / \mathrm{L}$ & $<40.0$ & $32.0(23.0-47.3)$ & $36.5(32.3-40.8)$ & $33.5(25.8-94.8)$ & 0.060 \\
\hline Albumin, g/L & $34.0-54.0$ & $34.2(30.5-38.1)$ & $28.3(27.2-32.0)$ & $30.4(29.0-32.3)$ & 0.008 \\
\hline Globulin, g/L & $20.0-35.0$ & $30.5(26.1-34.6)$ & $29.4(25.0-32.2)$ & $29.8(26.6-32.9)$ & 0.129 \\
\hline $\mathrm{LDH}, \mathrm{U} / \mathrm{L}$ & $109-245$ & $286(207-409)$ & $302(250-407)$ & $316(245-412)$ & 0.038 \\
\hline Creatinine, $\mu \mathrm{mol} / \mathrm{L}$ & $50.00-120.00$ & $68.00(55.50-81.35)$ & $76.35(56.43-87.43)$ & $64.50(54.18-110.15)$ & 0.090 \\
\hline $\mathrm{ESR}, \mathrm{mm} / \mathrm{h}$ & $<15.0$ & $46.2(25.3-62.5)$ & $42.0(19.3-58.8)$ & $49.0(25.0-77.8)$ & 0.160 \\
\hline $\mathrm{CRP}, \mathrm{mg} / \mathrm{L}$ & $<8.0$ & $13.2(2.9-61.7)$ & $44.4(39.8-146.1)$ & $31.4(16.9-96.0)$ & 0.011 \\
\hline Serum ferritin, $\mathrm{ng} / \mathrm{mL}$ & $21.80-275.0$ & $790.52(401.97-1324.91)$ & $1082.60(617.30-1339.50)$ & $762.72(533.98-1178.14)$ & 0.288 \\
\hline $\mathrm{IL}-6, \mathrm{pg} / \mathrm{mL}$ & $0.10-2.90$ & $9.36(6.52-13.80)$ & $9.10(5.44-34.27)$ & $9.62(6.51-13.47)$ & 0.194 \\
\hline $\mathrm{BNP}, \mathrm{pg} / \mathrm{mL}$ & $0.0-100.0$ & $62.9(26.6-115.8)$ & $73.0(32.4-253.5)$ & $73.2(30.0-127.7)$ & 0.877 \\
\hline D-dimer, ug/mL & $<0.50$ & $0.67(0.42-1.79)$ & $0.74(0.48-2.06)$ & $1.09(0.56-3.48)$ & 0.013 \\
\hline FIB, g/L & $2.00-4.00$ & $3.60(2.86-4.80)$ & $4.35(3.53-5.55)$ & $4.50(3.83-6.25)$ & 0.036 \\
\hline PT, s & $11.0-16.0$ & $11.3(10.6-12.2)$ & $11.5(10.1-12.5)$ & $11.5(10.0-12.5)$ & 0.127 \\
\hline APTT, s & $27.0-45.0$ & $28.2(25.0-32.5)$ & $30.8(26.5-33.6)$ & $22.3(19.3-32.2)$ & 0.004 \\
\hline
\end{tabular}

WBC white blood cell count, $N C$ neutrophil count, $L C$ lymphocyte count, $N L R$ neutrophil-to-lymphocyte ratio, $\mathrm{SaO} 2$ oxyhemoglobin saturation, $T B$ total bilirubin, $A L T$ alanine aminotransferase, $A S T$ aspartate aminotransferase, $L D H$ lactate dehydrogenase, $E S R$ erythrocyte sedimentation rate, $C R P$ c-reactive protein, $I L-6$ interleukin-6, BNP b-type natriuretic peptide, FIB fibrinogen, $P T$ prothrombin time, APTT activated partial thromboplastin time

Table 5 Outcome of multivariable logistic regression

\begin{tabular}{lrr}
\hline & OR $(95 \% \mathrm{CI})$ & $P$ value \\
\hline Severity as dependent variable & \\
Hypertension & $1.562(0.929-2.625)$ & 0.092 \\
Age, years & $1.049(1.030-1.067)$ & $<0.001$ \\
Female sex $(v s$ male $)$ & $0.523(0.319-0.856)$ & 0.010 \\
Mortality as dependent variable & \\
Hypertension & $1.262(0.683-2.332)$ & 0.458 \\
Age, years & $1.042(1.018-1.066)$ & $<0.001$ \\
Female sex $(v s$ male $)$ & $0.476(0.254-0.893)$ & 0.021 \\
\hline
\end{tabular}

OR odds ratio, $95 \%$ CI $95 \%$ confidence interval.

of IL-6, interleukin-7, granulocyte-macrophage colony-stimulating factor, and tumor necrosis factor $\alpha$. It is worth noting that previous clinical studies have found that the increase in these cytokines is also associated with the development of hypertension [13]. A notable example is that IL-6 has been observed to be closely related to the poor prognosis of patients with COVID-19 [14], which is also one of the important cytokines regulating immune-inflammatory responses in hypertension. In addition, in hypertensive patients with COVID-19, due to the imbalance in the renin-angiotensin system (RAS), the NADH/NADPH oxidase system may be activated by excessive inflammatory reactions or a large number of cytokines [15], resulting in cell damage and vasoconstriction [16], aggravating lung damage in COVID-19 and leading to a poor prognosis. The pathophysiology and inflammatory disorders of hypertension may partly explain poor prognoses for COVID-19. In addition, the effect of antihypertensive drugs on COVID-19 in patients with hypertension is controversial. On the one hand, the use of angiotensin-converting enzyme inhibitors (ACEIs) can result in a decrease in angiotensin-converting enzyme (ACE) and an increase in the expression of ACE2 in the lungs [17]. More ACE2 may mediate the invasion and infection of SARS-COV-2 into lung cells, resulting in viral spread and aggravation of symptoms; on the other hand, as a negative regulator of the RAS system, ACE2 can catalyze the hydrolysis of Ang II to Ang1-7, and Ang1-7 binds to the Mas receptor and plays an important role in antialveolar epithelial apoptosis, lowering blood pressure and protecting 
Table 6 Comparison of laboratory indices between hypertensive COVID-19 patients with and without other comorbidities

\begin{tabular}{|c|c|c|c|c|c|}
\hline & Normal range & Total $(n=113)$ & Noncomorbidity $(n=65)$ & Comorbidity $(n=48)$ & $P$ Value \\
\hline WBC, $\times 109 / \mathrm{L}$ & $4.00-10.00$ & $6.77(5.04-9.61)$ & $8.63(5.19-12.55)$ & $7.94(5.71-9.31)$ & 0.156 \\
\hline $\mathrm{NC}, \times 109 / \mathrm{L}$ & $1.80-6.30$ & $5.36(3.56-7.98)$ & $7.96(3.61-11.51)$ & $6.22(4.44-8.17)$ & 0.128 \\
\hline $\mathrm{LC}, \times 109 / \mathrm{L}$ & $1.10-3.20$ & $0.78(0.55-1.17)$ & $0.56(0.35-0.73)$ & $0.72(0.58-1.07)$ & 0.585 \\
\hline NLR & $<5.87$ & $6.36(2.99-14.59)$ & $15.77(4.16-33.17)$ & $8.26(4.49-13.04)$ & 0.148 \\
\hline $\mathrm{SaO} 2, \%$ & $>95.0$ & $94.5(89.0-97.0)$ & $87.0(85.0-96.6)$ & $93.8(87.0-97.7)$ & 0.071 \\
\hline $\mathrm{TB}, \mu \mathrm{mol} / \mathrm{L}$ & $5.10-19.00$ & $11.8(9.2-16.2)$ & $12.0(10.4-19.6)$ & $11.2(10.1-14.2)$ & 0.791 \\
\hline ALT, U/L & $<40.0$ & $33.0(19.0-57.5)$ & $56.0(26.0-128.0)$ & $69.5(35.8-107.3)$ & 0.160 \\
\hline AST, U/L & $<40.0$ & $34.5(24.0-56.3)$ & $40.0(31.0-78.0)$ & $54.5(48.0-80.5)$ & 0.740 \\
\hline Albumin, $\mathrm{g} / \mathrm{L}$ & $34.0-54.0$ & $31.9(29.4-35.5)$ & $30.8(27.0-31.9)$ & $35.7(30.8-38.3)$ & 0.502 \\
\hline Globulin, g/L & $20.0-35.0$ & $31.7(28.3-35.7)$ & $31.9(29.4-35.9)$ & $30.7(28.9-33.6)$ & 0.331 \\
\hline $\mathrm{LDH}, \mathrm{U} / \mathrm{L}$ & $109-245$ & $306(217-429)$ & $424(306-497)$ & $331(243-459)$ & 0.350 \\
\hline Creatinine, $\mu \mathrm{mol} / \mathrm{L}$ & $50.00-120.00$ & $72.50(60.70-95.18)$ & $72.30(54.20-110.40)$ & $92.05(70.18-151.88)$ & 0.167 \\
\hline $\mathrm{ESR}, \mathrm{mm} / \mathrm{h}$ & $<15.0$ & $49.0(35.3-69.0)$ & $55.0(37.0-89.0)$ & $56.5(39.3-72.5)$ & 0.962 \\
\hline $\mathrm{CRP}, \mathrm{mg} / \mathrm{L}$ & $<8.0$ & $35.3(6.1-124.3)$ & $68.0(24.8-180.0)$ & $81.0(26.7-145.2)$ & 0.548 \\
\hline Serum ferritin, $\mathrm{ng} / \mathrm{mL}$ & $21.80-275.0$ & 778.27 (463.77-1301.59) & $1101.76(680.05-2100.00)$ & 915.17 (413.27-1437.52) & 0.726 \\
\hline IL-6, pg/mL & $0.10-2.90$ & $10.43(7.05-13.71)$ & $11.64(7.11-12.41)$ & $10.54(9.41-14.60)$ & 0.636 \\
\hline BNP, $\mathrm{pg} / \mathrm{mL}$ & $0.0-100.0$ & $72.5(31.1-174.3)$ & $72.5(31.1-75.5)$ & $51.2(28.7-126.5)$ & 0.047 \\
\hline D-dimer, ug/mL & $<0.50$ & $0.99(0.55-3.37)$ & $1.48(0.69-2.67)$ & $0.90(0.35-2.89)$ & 0.369 \\
\hline $\mathrm{FIB}, \mathrm{g} / \mathrm{L}$ & $2.00-4.00$ & $4.00(2.99-5.15)$ & $4.90(4.10-6.20)$ & $5.25(3.63-7.70)$ & 0.537 \\
\hline PT, s & $11.0-16.0$ & $11.3(10.4-12.2)$ & $11.1(10.2-12.5)$ & $11.4(10.4-12.4)$ & 0.320 \\
\hline APTT, s & $27.0-45.0$ & $26.5(23.6-31.5)$ & $23.4(20.0-30.2)$ & $25.1(23.5-31.7)$ & 0.635 \\
\hline
\end{tabular}

$W B C$ white blood cell count, $N C$ neutrophil count, $L C$ lymphocyte count, $N L R$ neutrophil-to-lymphocyte ratio, $\mathrm{SaO} 2$ oxyhemoglobin saturation, $T B$ total bilirubin, $A L T$ alanine aminotransferase, $A S T$ aspartate aminotransferase, $L D H$ lactate dehydrogenase, $E S R$ erythrocyte sedimentation rate, $C R P$ c-reactive protein, $I L-6$ interleukin-6, $B N P$ b-type natriuretic peptide, $F I B$ fibrinogen, $P T$ prothrombin time, APTT activated partial thromboplastin time

the endothelium [18]. By reducing the effect of Ang II and increasing the production of Ang1-7, ACE2 can induce vasodilation and an antiinflammatory response [19], which helps to reduce the degree of lung injury and prevent the emergence of acute respiratory distress syndrome (ARDS) and acute lung injury. A study from China involving 1128 hypertensive COVID-19 patients showed that hypertensive patients who used ACEIs or angiotension receptor blocker (ARBs) in hospitals had a lower risk of all-cause death than those who did not use ACEIs/ARBs [20]. In addition, an article from the COVID-19 working group of the European Society of Hypertension pointed out that there is no evidence to support that RAS inhibitors are harmful to COVID-19 patients, so there is no reason for hypertensive patients with stable conditions to stop using RAS inhibitors [21].

Our multivariate analysis showed that after adjusting for age and sex, hypertension did not show a significant correlation with increased COVID-19 disease severity or mortality, which was contrary to the results of a previous large-sample study [20]. We speculate that the possible reasons are that the sample size of our data is small, the number of end events is small, and the test efficiency is insufficient; at the same time, the regression models used by both studies are different, and the adjusted variables are also different. At present, there is a lack of research on hypertensive COVID-19 patients, especially those adjusted for age and sex, and our study can be regarded as a reference.

Overall, our comparative analysis of COVID-19 patients with and without hypertension showed that hypertensive patients had greater disease severity and adverse progression than nonhypertensive patients. However, this result may be related to the higher age in this group than in nonhypertension group. In addition, there were more severe symptoms; greater likelihood of other complications, such as diabetes; more likelihood of transfer to the ICU; and worse laboratory indicators. COVID-19 patients with hypertension need additional attention to prevent adverse outcomes.

Our study has the following limitations. First, the number of patients included in our survey is not enough, and the effectiveness of the testing may not be sufficient; in future studies, we need a larger sample size of clinical data to support our conclusions. Second, we did not analyze complications such as acute renal injury and ARDS because we focused on analyzing the risks associated with hypertension; in addition, due to the retrospective nature of this 
study, as well as the urgency of time and difficulties in collection, there was a lack of the use of antihypertensive drugs in patients. Third, because the diagnosis of hypertension was extracted from medical history data, there may be a very small number of patients with hypertension, which may not have been accurately recorded. However, these limitations will not affect the reliability of our overall results.

Acknowledgements We thank all patients involved in the study.

\section{Compliance with ethical standards}

Conflict of interest The authors declare that they have no conflict of interest.

Ethical approval All procedures performed in studies involving human participants were in accordance with the ethical standards of the institutional and/or national research committee and with the 1964 Helsinki declaration and its later amendments or comparable ethical standards.

Informed consent Written or oral informed consent was waived.

Publisher's note Springer Nature remains neutral with regard to jurisdictional claims in published maps and institutional affiliations.

\section{References}

1. Phelan AL, Katz R, Gostin LO. The Novel Coronavirus Originating in Wuhan, China: Challenges for Global Health Governance. JAMA. 2020;323:709-10.

2. Zhou F, Yu T, Du R, Fan G, Liu Y, Liu Z, et al. Clinical course and risk factors for mortality of adult inpatients with COVID-19 in Wuhan, China: a retrospective cohort study. Lancet. 2020;395:1054-62.

3. Chan JW, Ng CK, Chan YH, Mok TY, Lee S, Chu SY, et al. Short term outcome and risk factors for adverse clinical outcomes in adults with severe acute respiratory syndrome (SARS). Thorax. 2003;58:686-9.

4. Distribution of novel coronavirus pneumonia. 2020 [cited 2020 4/ 20]; Available from: http://2019ncov.chinacdc.cn/2019-nCoV/ global.html.

5. Zhou P, Yang XL, Wang XG, Hu B, Zhang L, Zhang W, et al. A pneumonia outbreak associated with a new coronavirus of probable bat origin. Nature. 2020;579:270-3.
6. Hamming I, Timens W, Bulthuis ML, Lely AT, Navis G, van Goor H. Tissue distribution of ACE2 protein, the functional receptor for SARS coronavirus. A first step in understanding SARS pathogenesis. J Pathol. 2004;203:631-7.

7. Huang C, Wang Y, Li X, Ren L, Zhao J, Hu Y, et al. Clinical features of patients infected with 2019 novel coronavirus in Wuhan, China. Lancet. 2020;395:497-506.

8. Guan WJ, Ni ZY, Hu Y, Liang WH, Ou CQ, He JX, et al., Clinical Characteristics of Coronavirus Disease 2019 in China. N Engl J Med. 2020;382:1708-20.

9. National Health Commission of the People's Republic of China home page. Available from: http://www.nhc.gov.cn.

10. Wang ZW, Chen Z, Zhang LF, Wang X, Hao G, Zhang ZG, et al. Status of hypertension in China results from the China hypertension survey, 2012-2015. Circulation. 2018;137:2344-56.

11. Morra ME, Van Thanh L, Kamel MG, Ghazy AA, Altibi AMA, Dat LM, et al. Clinical outcomes of current medical approaches for Middle East respiratory syndrome: a systematic review and meta-analysis. Rev Med Virol. 2018;28:e1977.

12. Matsuyama R, Nishiura H, Kutsuna S, Hayakawa K, Ohmagari N. Clinical determinants of the severity of Middle East respiratory syndrome (MERS): a systematic review and meta-analysis. BMC Public Health. 2016;16:1203.

13. Drummond GR, Vinh A, Guzik TJ, Sobey CG. Immune mechanisms of hypertension. Nat Rev Immunol. 2019;19:517-32.

14. NHCotPsRo, C. Chinese Clinical Guidance for COVID-19 Pneumonia Diagnosis and Treatment, 7th ed. 2020; http://kjfy. meetingchina.org/msite/news/show/cn/3337.html.

15. Zhang Y, Murugesan P, Huang K, Cai H. NADPH oxidases and oxidase crosstalk in cardiovascular diseases: novel therapeutic targets. Nat Rev Cardiol. 2020;17:170-94.

16. Dhaun N, Webb DJ. Endothelins in cardiovascular biology and therapeutics. Nat Rev Cardiol. 2019;16:491-502.

17. Soler MJ, Barrios C, Oliva R, Batlle D. Pharmacologic modulation of ACE2 expression. Curr Hypertension Rep. 2008;10:410-4.

18. Imai Y, Kuba K, Penninger JM. The discovery of angiotensinconverting enzyme 2 and its role in acute lung injury in mice. Exp Physiol. 2008;93:543-8.

19. Oparil S, Schmieder RE. New approaches in the treatment of hypertension. Circ Res. 2015;116:1074-95.

20. Zhang P, Zhu L, Cai J, Lei F, Qin JJ, Xie J, et al., Association of inpatient use of angiotensin converting enzyme inhibitors and angiotensin II receptor blockers with mortality among patients with hypertension hospitalized with COVID-19. Circ Res 2020. https://doi.org/10.1161/CIRCRESAHA.120.317134). [Epub ahead of print].

21. Kreutz, R, Algharably EAE, Azizi M, Dobrowolski P, Guzik T, Januszewicz A, et al., Hypertension, the renin-angiotensin system, and the risk of lower respiratory tract infections and lung injury: implications for COVID-19. Cardiovasc Res, cvaa097. https://doi. org/10.1093/cvr/cvaa097. 